\title{
Fluid dynamic-based Engineering design of a Full-Scale Device for the improvement of Extra Virgin Olive Oil Yield and Quality by means of Combined Acoustic Cavitation and Thermal Conditioning
}

\author{
Riccardo Amirante ${ }^{1, *}$, Elia Distaso $^{1}$, Paolo Tamburrano ${ }^{1}$, Filomena Faustina Rina Corbo $^{2}$, \\ Giuseppe Calò ${ }^{1,3}$, and Maria Lisa Clodoveo ${ }^{3}$ \\ ${ }^{1}$ Department of Mechanics, Mathematics and Management (DMMM), Politecnico di Bari, via E. \\ Orabona 4, 70126 Bari, Italy \\ ${ }^{2}$ Department of Pharmacy-Pharmaceutical Sciences, University of Bari “Aldo Moro", Via E. Orabona \\ 4, 70126 Bari, Italy \\ ${ }^{3}$ Interdisciplinary Department of Medicine, University of Bari “Aldo Moro", Piazza Giulio Cesare, \\ 11, 70124 Bari, Italy
}

\begin{abstract}
After some hesitations, the scientific community is jointly converging on the benefits due to the ultrasound treatment by means of mechanical effects generated by acoustic cavitation phenomena occurring into the olive oil paste proposed initially by Amirante and Clodoveo. In recent works, many authors have now confirmed that this promising emerging technology produces relevant beneficial effects if applied to the extraction process under well-controlled conditions. In the last years, the industrial applications of ultrasound (US) in the Extra-Virgin Olive Oil (EVOO) extraction process are changing the paradigm of the knowledge in this field of interest due to a great effort of the research activity. In the present work, the design of the device by means a Three-Dimensional (3D) Multiphase Computational Fluid Dynamic (CFD) analysis was performed, which describes the ultrasound effects in the olive paste, necessary to control the US waves propagation. Thus, fluid dynamic analysis allowed to predict the flow path in the ultrasound devices, to evaluate the flow parameters of the olive paste inside the SHE and the cavitation phenomenon, with the aim to find an optimal design, capable to ensure the best ultrasounds and mixing effects. Moreover, experimental results demonstrated that the machine can guarantee an actual simultaneous improvement of the olive oil extraction yield, as well as of the product quality. Finally, the results from sensory evaluations are summarized confirming the goodness of EVOO obtained by means of US.
\end{abstract}

\footnotetext{
* Corresponding author: amirante@poliba.it
} 


\section{Introduction}

Ultrasound (US) is a promising emerging technology that has already found application in the food industry [1-3] due to its significant effects on the processes, such as higher product yields, shorter processing times, reduced operating and maintenance costs, improved taste, texture, flavour and colour [4]. Nowadays many other authors show how US has promising application in the field of virgin olive oil industry, due to the mechanical and thermal effects useful to guarantee adequate oil yields, thus reducing the process time and improving the process efficiency [5-7], confirming the strategy proposed in this paper. The thermal effect occurs when kinetic energy of the ultrasound waves is converted into the thermal energy due to the turbulence increment in the matter $[8,9]$.

The full scale application of ultrasounds in the Extra-Virgin Olive Oil (EVOO) extraction process is more recent but it appears very promising due to the interesting industrial scale application proposed by Amirante and Clodoveo in the last years [10], summarized in a recent paper by Clodoveo [11], such as by the same Bejaoui, Jimenez, Beltran, Patist, et al. $[4,7,12,13]$. This new technology exploits the simultaneous effects of the ultrasounds and heat exchange (the so called Sono-Heat-Exchanger, SHE) and it is not only relevant for the enhancements in the extraction yields [14], but also from an economical point of view for the skill to improve phenols contents [15] to guarantee the possibility of using health claims $[16,17]$.

The main effect of US technique on the olive paste is due to the mechanical effect of the acoustic cavitation phenomena. The effect of acoustic cavitation promotes the breaking of tissue structures, including membranes of elaioplasts freeing the trapped oily phase $[18,19]$. These phenomena make that the application of ultrasound to the extraction process of EVOO enhances the release of oil and minor compounds [20]. Another important consideration is that ultrasounds increase the kinetic of the olive paste promoting the coalescence phenomena, enhancing the probability of particles collision. So that the amount of oil recoverable during the centrifugation phase can be substantially increased [21] and the malaxation processing time drastically reduced, which makes possible to think that malaxation could be completely eliminated by realizing an actual fully continuous process. Another property of low frequencies ultrasounds $(<30 \mathrm{kHz})$ is the possibility of splitting the emulsion into its component, aqueous and oily phases [18], improving the extraction process.

As consequence, the research efforts of Amirante and Clodoveo in designing the SonoHeat-Exchanger (SHE) have produced an advanced machine able to perform three fundamental goals: transform extraction process into a fully continuous phase, increase yield, increase quality $[6,21,22]$. However, the design of SHE is far from easy, due to the absence of calculation tools able to predict the ultrasounds propagation into the liquid, especially if the ultrasound sources are close to each other, as in the SHE machine. In the design process, some crucial points must be taken into account: the specific energy to be provided, the heat exchange surface area, the cross-section shape and the relative position of the US transducers. Regarding the specific energy, it was established by means of experimental tests proposed by Clodoveo in 2013 [5] and it is useful to define the power of machine [6,10].

This paper shows how can be designed the heat exchanger, such as the SHE cross section and its shape, by means of a Three-Dimensional (3D) Multiphase Computational Fluid Dynamic (CFD), used as auxiliary tool. And, in particular, both steady-state and transient simulations were performed with the aim of defining the cross section of the SHE that ensures the best performance, borrowing optimization systems applied to other machines design [23]. In addition, these calculations allowed to monitor the flow parameters able to influence the 
process, avoiding expensive and time-consuming preliminary experimental tests. Once designed, the SHE was collocated between the crusher and the decanter of an existing plant and experimental tests were carried out. The increase in values recorded for both the extraction yield and the phenols contents demonstrated the relevance of this innovation, confirming the significant progress made towards the transformation of the malaxing step from a batch operation into an actual continuous process.

\section{Material and Methods}

Fig. 1 shows a typical so-called "continuous" process where the harvested olives are moved to the extraction plant and then the olives are dropped into a hopper and laid down onto a conveyor belt that carries them to the washing machines. A vibrating machine (1) removes leaves and other debris to protect the extraction plant and avoid the off flavours deriving from the presence of foreign bodies. After that, the olives are also washed (2) to remove soil or other residues and so the pre-process stage is finished. Now another conveyor belt carries the olives to the next phase, the crushing (3) which is the first phase of the extraction process. The crushing phase breaks the olive fruit tissues by means of a strong mechanical action, which breaks up the vegetal cells, producing the release of oil, as well as heat due to energy dissipation. The olive paste obtained is moved to the following stage by means of a piping and an upstream mono pump (4). Usually, malaxation (5) represents the subsequent step and it consists of a usual cylindrical tank equipped with a shaft with rotating arms and stainless steel blades, while in the new concept of the proposed layout, the ultrasound treatment is applied by means of the SHE (8).

\section{Fluid Dynamic-based Engineering design of the SHE}

The finite-volume method was used to discretize and solve the flow by employing the commercial software Fluent 17.1, manufactured by Ansys. The flow was modelled as laminar, due to the high viscosity of the olive paste, which remains laminar even at large flow rates [24]. The olive oil paste was modelled as a non-Newtonian fluid [6] and it was defined as compressible-liquid following the Tait equation [25] and having a density of $1126 \mathrm{~kg} / \mathrm{m}^{3}$ at standard conditions [6], a bulk modulus of $1.6 \mathrm{e}+09$ and a density exponent equal to 11 . For the transient simulations, in order to simulate the unsteady cavitating flow, the Schnerr model was adopted [26] and, specifically, a bubble number density equal to $1 \mathrm{e}+09$ and a vapour pressure of $3540 \mathrm{~Pa}$ were set, according with the water value at the working temperature.

It was possible to drastically reduce the overall computational time by taking advantage of the axial symmetry of the device geometry. Therefore, it was sufficient to discretise only a quarter of the whole computational domain, comprising two sides of the octagonal prism, as highlighted in the right part of Fig. 2. It was considered that two rows of transducers placed on two adjacent lateral faces of the octagonal prism were staggered each other by half of the longitudinal distance between two transducers. A whole structured dynamic grid was used to mesh a quarter of the geometry, in order to reduce the overall computational (left part of Fig. 2). A grid independence analysis was performed to properly select the grid size. Four computational grids were generated, namely, with 1560000 cells (grid 1), 975000 cells (grid 2), 465000 cells (grid 3), 282000 cells (grid 4). The pressure drops calculated at the last time step was used to compare the four grids. Among the four computational grids, "grid 2" was selected since it provides the best compromise between accuracy and computational time. 


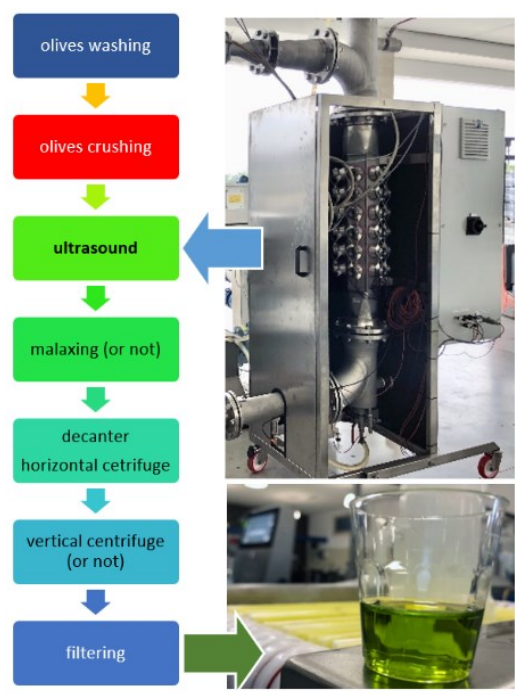

Fig. 1 Evoo modified scheme proposed in the present work. On the right a picture of the Sono-HeatExchanger (SHE) system (2019 version).

The specific energy generated by the ultrasound transducers was set equal to the optimal value obtained from a preliminary analysis carried out in static conditions on small specimens $[5,6,18]$. From this consideration and for a total installed power of $5.5 \mathrm{~kW}$, it was possible to estimate that $1500 \mathrm{~kg} / \mathrm{h}$ represents the value of mass flow rate that gives the best value of specific energy $(15 \mathrm{~kJ} / \mathrm{kg})$. This value of the mass flow rate was imposed at the "flow inlet" (lower green arrow Fig. 2 right part) boundary of the domain in the simulations (green surface in Fig. 2 left part). A static pressure of 2 bar (absolute pressure) was set at the "flow outlet", according to the pressure drop estimated to push the olive paste into the malaxer of the experimental plant. This pressure condition has been experimentally verified during the experimental tests. Fixed temperatures were assigned to the non-moving walls. In the same figure the oscillating surfaces under each ultrasound transducer are visible. A periodic alternative motion was assigned to these surfaces. An oscillation frequency of $23 \mathrm{kHz}$ was imposed, according to that of the transducer plates employed in the experiments. Axialsymmetric periodic conditions were assigned to the non-solid remaining boundaries of the computational domain.

\section{Results and Discussion}

\subsection{Fluid Dynamic Analysis}

To understand the numerical results, Fig. 3 shows the location of two points used in the analysis of the transient simulations and a schematic indication of the transducer motion is reported above each graph.

Referring to Fig. 4, the pressure starts to increase as the transducer surface moves down, in the first hemicycle. When the surface reverses its route and starts moving up, the pressure suddenly decreases until it reaches the vapour pressure limit, leading to the onset of the vapour bubbles denoted by the increase of the vapour volume fraction. Once the cavitation 
bubbles reach a critical size, they abruptly implode, making the vapour fraction decreasing accordingly. Fig. 4 shows that more than one cycle was necessary for making this happening. From this result can be stated that, in a given point, cavitation occurs with a periodicity that does not necessarily match the transducers oscillation frequency.

This behaviour can be reasonably explained by considering that the pressure waves produced by a single transducer are not free to propagate in the liquid medium, but they experience many complex interactions with other negative or positive pressure waves generated both by wall reflections and other adjacent transducers. Therefore, the interaction between the oscillating surfaces and the non-Newtonian fluid produces the instauration of a complex flow field having an own temporal periodicity. In fact, after the transient initial phase is extinguished, after five complete cycles, it is possible to observe from Fig. 4 that the pressure trace assumes a profile between the $5^{\text {th }}$ and the $9^{\text {th }}$ cycle that looks pretty similar to that observable between the $9^{\text {th }}$ and the $13^{\text {th }}$ cycle. This suggests that a pseudo-stationary condition has been established and, from this point forward, the space distribution of a fluid dynamic variable will look similar if compared to others taken at a temporal distance of $1.74 \mu \mathrm{s}$, namely 4 complete cycles.
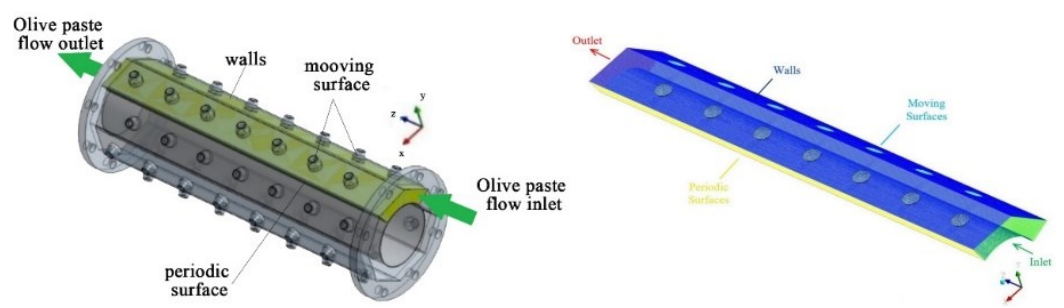

Fig. 2 Modelled domain of the Sono-Heat-Exchanger (SHE). Computational full-structured dynamic mesh used to discretize the fluid domain of interest of the Sono-Heat-Exchanger (SHE).

This appears even more evident by comparing the pressure histories in two different points, as proposed in Fig. 5, in which the additional purple trace refers to the purple probe point previously shown in Fig. 3. Fig. 5 neglects the initial transitory and considers the events comprised between the $5^{\text {th }}$ and the $13^{\text {th }}$ cycle. Despite the pressure fluctuations differ a little from each other and appear slightly shifted with time one from the other, it is possible to clearly observe that they preserve the same periodicity. The small differences are simply due to the fact that the two points were located at different distances from the closer transducer. In this specific case, i. e. at this frequency and for the considered operating conditions and geometries, four complete oscillations of the transducers are needed in order to complete a cavitation cycle.

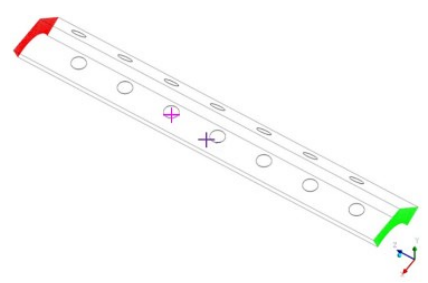

(a)

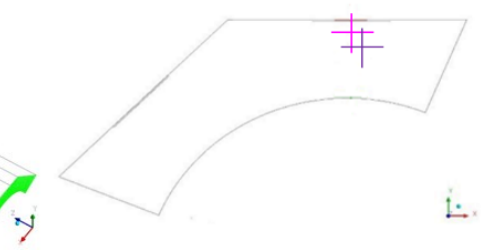

(b)

Fig. 3 Location of two points used in the analysis of the transient simulations. Isometric (a) and frontal (b) views. 


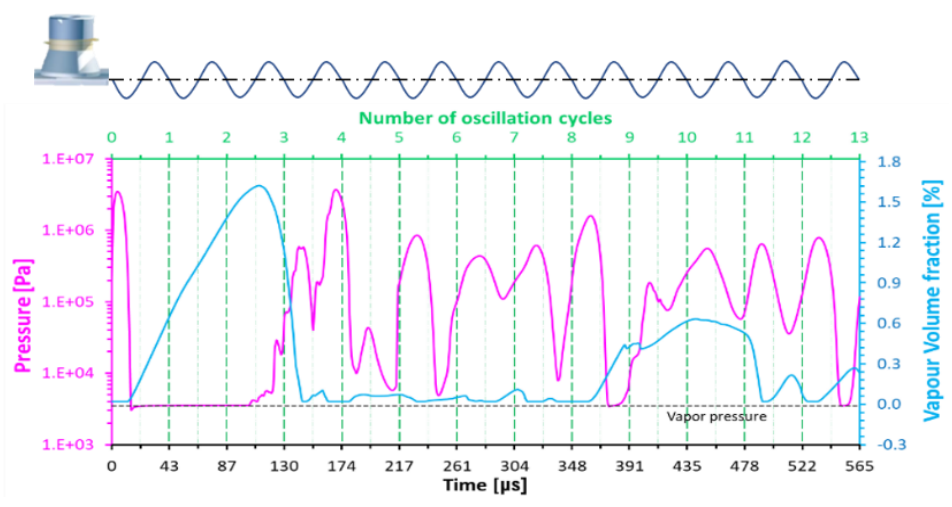

Fig. 4 Pressure and vapour volume fraction evolution with time recorded at the pink point of Fig. 3. A schematic indication of the transducer motion is reported above the graph.

A crucial result that it was possible to obtain from these simulations was the definition of the optimal height for the SHE cross-section. The choice was based on the consideration that the optimal cross-section should represent the best compromise between the need of maximizing the ultrasound effects and that of avoiding undesired interference effects between adjacent transducers [14]. The former objective can be achieved by reducing as much as possible the height of the SHE cross-section, for ensuring that cavitation can be effectively promoted up to the opposite surface of the SHE. However, a too narrow crosssection can induce a high pressure drop, that would make the device not energetically convenient, besides creating the conditions for inhibiting the ultrasound beneficial effects.

Fig. 6 reports the pressure evolution with time recorded in three different points belonging to a straight line normal to the moving surface of a central transducer and passing through the centre of it, as illustrated in Fig. 7. The probe points are equally spaced one from each other, with the blue one located in the middle of the cross-section height. The red point was located extremely close to the transducer surface and allowed to appreciate the most intense ultrasound effects. The green point was located close to the opposite surface with the aim of monitoring how deeply the cavitation can occur, which, in turn, gives a direct information about the effectiveness of the final design of the SHE.

At the beginning of the simulation the transducer surface starts to move down, and a pressure peak is recorded near the transducer surface. The generated pressure wave then propagates radially, until it reaches the opposite wall. This is inferable from the graph of Fig. 6 , by noting that, in the first cycle, a pressure peak first appears in the red trace and then involves the blue and green traces, respectively, appearing gradually smothered, though. This shows that the present approach allows to monitor the pressure wave propagation and the correlated damping phenomena occurring within the non-Newtonian fluid. In the same way, it is possible to infer when and where cavitation is occurring, just by looking for those instants in which the pressure traces cross the vapour pressure threshold. This happens many times during the simulation, especially when the pseudo-stationary conditions are established. The most relevant result is that cavitation is recorded at each point of the cross-section since all the three lines reach the vapour pressure limit. This is therefore true also for the point placed on the opposite surface (green line in Fig. 6) on which, although such a limit is reached less times in comparison to the other two locations, the appearance of cavitation remains still appreciable. 


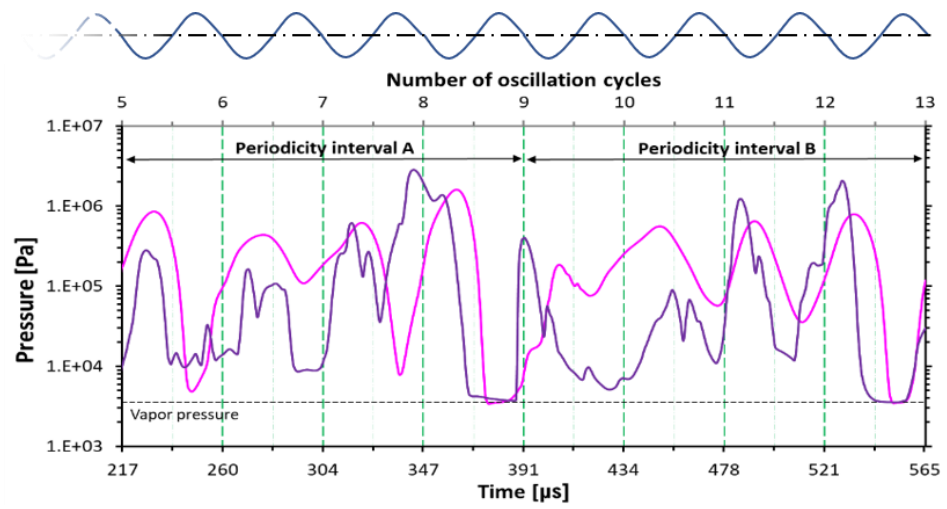

Fig. 5 Comparison between the pressure traces recorded at the two monitoring points of Fig. 3. A schematic indication of the transducer motion is reported above the graph.

Since there is no guarantee that what was observed locally can be generalized, it was chosen to extend the analysis to the whole domain by employing three control lines, aligned with the flow direction, as shown in Fig. 8, and passing through the three points of Fig. 7. The results deriving from this thorough additional analysis are reported from Fig. 9 to Fig. 11.

The solution obtained from the steady-state calculations was used as initial condition for the transient simulation. Therefore, at the starting point (upper left corner of Fig. 9) the pressure along the three control lines was exactly the same and appears almost constant in the space. Actually, a closer look at the frame taken at $t=0 \mu \mathrm{s}$, shows that each of the three pressure traces has a slight slope, accounting for the pressure drop due to friction along the length of the device discussed previously.

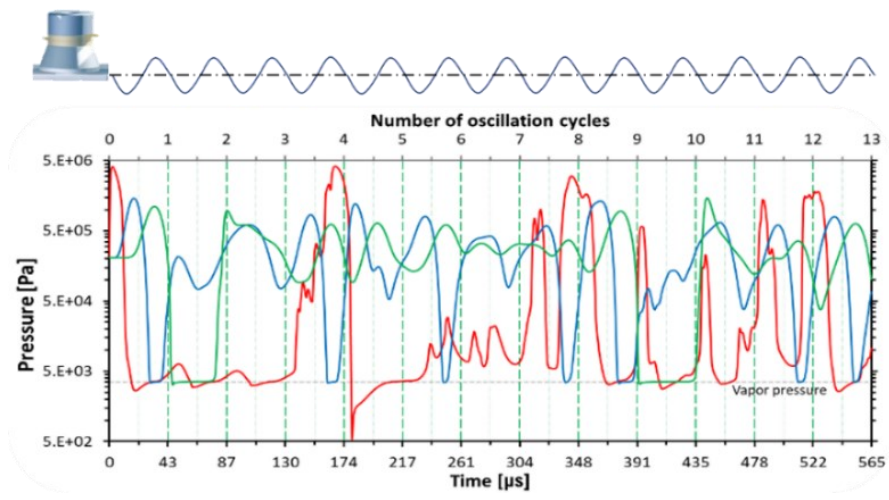

Fig. 6 Comparison between the pressure traces recorded at the three monitoring points of Fig. 7. A schematic indication of the transducer motion is reported above the graph.

When the transducers start to move, what was previously observed locally, it is also possible to observe globally, for each transducer, along the axial length of the device. Namely, from Fig. 4, it has been possible to affirm that the interaction between the oscillating surfaces and the non-Newtonian fluid produces the instauration of a complex flow field having an own temporal periodicity. Thus, in a given point, cavitation occurs with a periodicity that does not necessarily match the transducers oscillation frequency. 


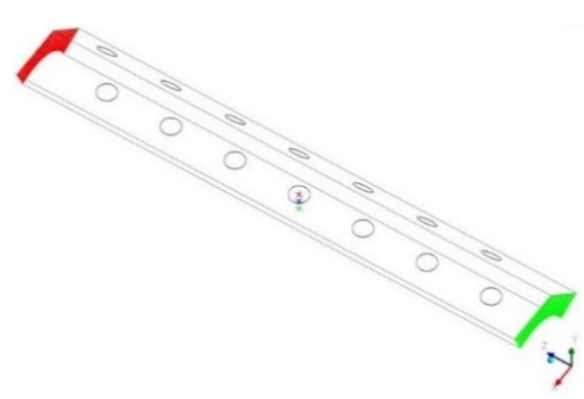

(a)

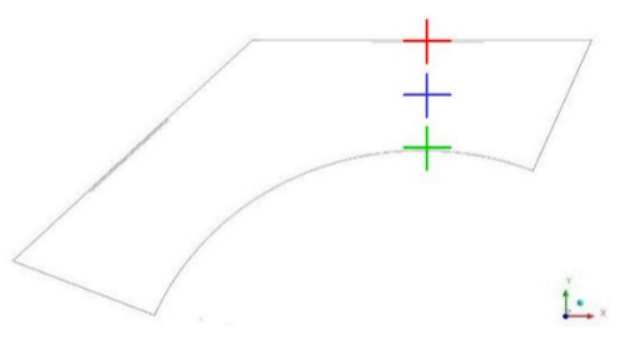

(b)

Fig. 7 Location of the three points used in the analysis of the transient simulations for studying the pressure evolution on a cross-section of the SHE. Isometric (a) and frontal (b) views.

Extending globally, compression waves are generated by the downwards movement of the transducer surfaces (red line at $t=1.21 \mu \mathrm{s}$ in Fig. 9). Such a wave pattern propagates normally to the flow direction, and when it is at mid-height of the cross-section (blue line at $t=21.74 \mu \mathrm{s}$ in Fig. 9), the moving surfaces have already reversed the route and vapour bubbles are creating near the transducers (red line at $t=21.74 \mu \mathrm{s}$ in Fig. 9). At the end of the first cycle the expansion wave generated by the upwards movement of the surfaces allows the pressure to go below the vapour pressure limit also at mid-height of the cross-section (blue line at $t=32.61 \mu \mathrm{s}$ in Fig. 9). At this time, the compression wave has reached the opposite wall of the device (green line at $t=32.61 \mu \mathrm{s}$ in Fig. 9). Only at the half of the second cycle favourable conditions for the generation of cavitation bubbles on the opposite wall are established. In the meantime, the other control lines start to record more complex wave interactions.

Fig. 10 shows the ending part of the initial transient stage, namely it shows salient phases going from the $3^{\text {rd }}$ to the $5^{\text {th }}$ cycle. The pressure traces start to assume less regular shapes, indicating that a deeper analysis is needed for an effective ultrasound treatment design.

Fig. 10 highlights that when a transducer surface moves alternatively up and down it superimposes to the flowing liquid a motion similar to that observable when a thrown stone plops into water producing waves and rings on calm and smooth water surface. Observing the shapes assumed by the red line between the $3^{\text {rd }}$ and the $4^{\text {th }}$ cycle, it is possible to see that each wave crest (or trough) has been spitted in three (o more) parts. This means that cavitation does not necessarily occur uniformly under the transducer surfaces. Namely, during the $3^{\text {rd }}$ cycle the pressure reached the vapour limit only in the central part of the moving surfaces, while at the half of the $4^{\text {th }}$ cycle $(t=152.17 \mu \mathrm{s}$ in Fig. 10), vapour is formed only near the periphery of the circular transducer surface. This has been recorded for the other two control lines, as well. This means that the zones interested by cavitation do not necessarily match the projection of the transducer surface. The cavitation points will differ very likely in size and in number with respect to the transducer surfaces, as it is possible to infer from the last part of the $5^{\text {th }}$ cycle reported in Fig. 10. From these observations it derives that the choice of a non-appropriate spacing between the transducers, as well as a wrong relative distribution of them, might probably result in undesirable interfering effects. On the other hand, one could take advantage of this aspect in the design process by exploiting the induced motion in the fluid to enhance the effectiveness of the ultrasound treatment, as shown later in the present analysis. 


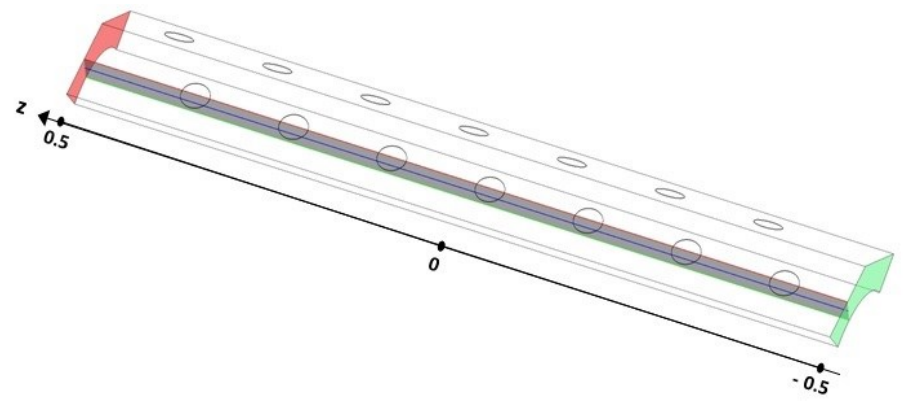

Fig. 8 Location and orientation of the control lines used in the analysis of the transient simulations for studying the pressure evolution along the axial direction of the SHE.

Once pseudo-stationary conditions are reached, all the observations made so far remain true. Fig. 11 compares the pressure distributions observable in frames taken at a temporary distance equal to one period. On the left-hand side of Fig. 11 there are four frames taken from the periodicity interval " $A$ " (as it was defined in Fig. 5) while on the right-hand side of Fig. 11 there are the corresponding frames belonging to the periodicity interval " $\mathrm{B}$ ", viz., taken four cycles later than the first ones.
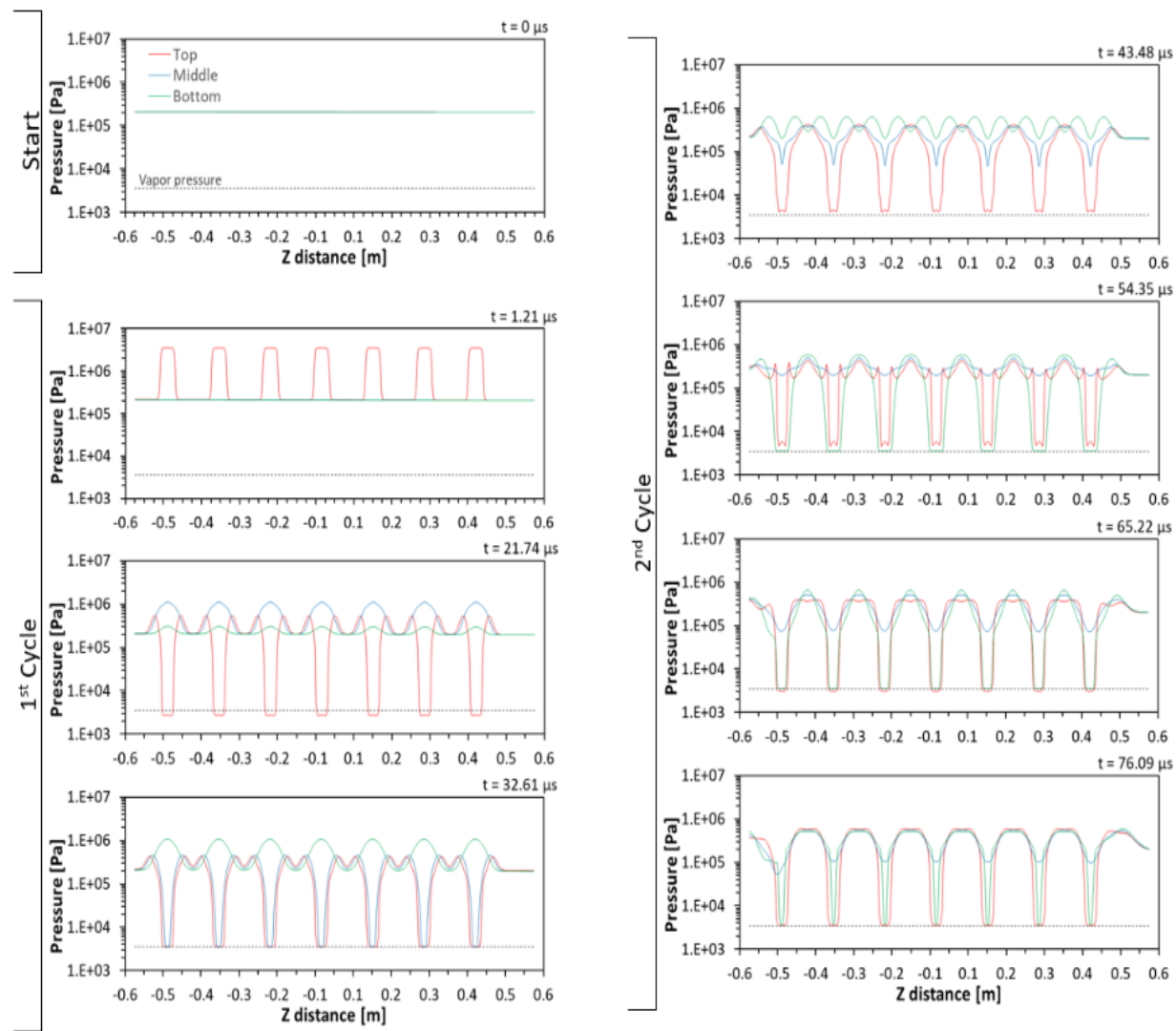

Fig. 9 Pressure traces recorded along the three control lines of Fig. 8 during the transient simulation. Initial solution and first two cycles. 
Fig. 11 highlights that similar pressure fields can be observed at corresponding times in two consecutive periods. For instance, in the first frame, both graphs show that large pressure oscillations can be observed only near the transducers, with the most pronounced ones located at the extremities of the device. In the second frame, both graphs show that vapour is creating at the middle of the cross-section height, while in the vicinities of the transducer surfaces this only happens near the inlet and the outlet sections. These results allow to observe that the transducers located near the entrance and the exit sections experience a different interaction with the flow, if compared with those located at mid-height of the device. This is an additional reason that explains why, after the transient, the pressure traces appear really different to those depicted in Fig. 9 and, consequently, the resulting flow much more complex.

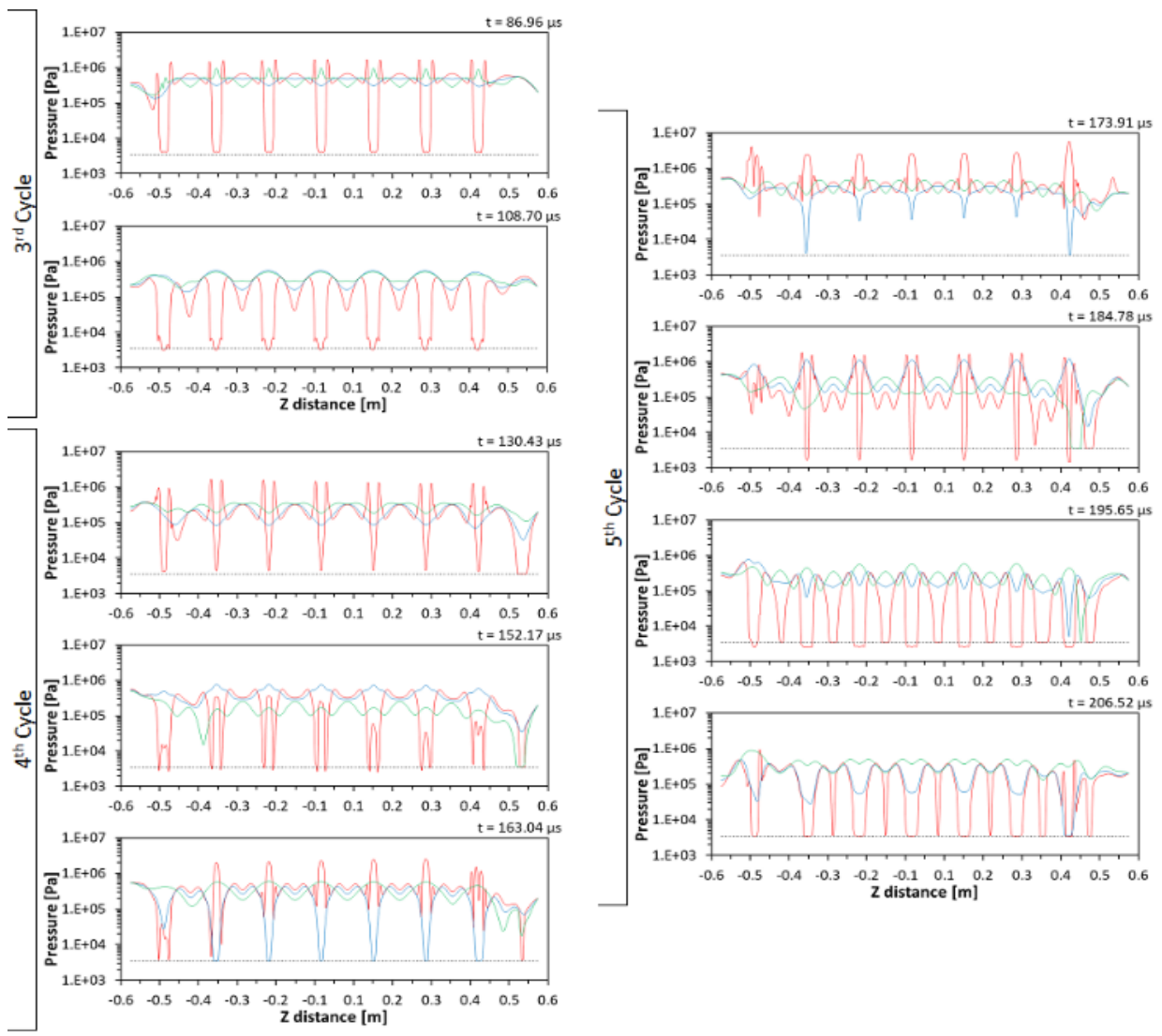

Fig. 10 Pressure traces recorded along the three control lines of Fig. 8 during the transient simulation. Final stages of the initial transient phase.

At conclusion of this analysis, the study of the flow field is proposed. For this purpose, four control lines are used to plot the profiles of the velocity components along the axial (zaxis in Fig. 12) and the circumferential (x-axis in Fig. 12) directions of the SHE. The lightblue and the purple control lines are thought to monitor what happens in the vicinities of a central transducer, while the pink and the yellow ones allow to analyse the flow between a couple of transducers. The undisturbed velocity profile does not have other components except for that along the axial direction (z). Namely, when the transducer surfaces are not moving, the olive paste flows through the SHE with a purely axial velocity having the profile 
depicted in Fig. 13 (a). In this case, the velocity varies parabolically along the height of the SHE cross-section (y-axis in Fig. 12), indicating that the flow is laminar and possesses a velocity magnitude in the order of a centimetre per second. It must be noted that the values on the y-axis have been made dimensionless.

Fig. 13 (b) reports the profiles of the circumferential velocity recorded on the four control lines at four different instants. These four times were chosen because representative of the main results obtained from the present analysis. Fig. 13 (c) reports, for the same instants, the profiles of the axial velocity recorded on the four control lines of Fig. 12. On Fig. 13, a graphical information about the location of the moving surface at that specific time is also reported for each of the four selected frames. The graphs of Fig. 13 (c) show that a nonnegligible momentum is transferred to the fluid by the transducers. At the end of an expansion stroke $(t=293.5 \mu s)$ the fluid is sucked up by the upwards movement of the transducer surface, as it is possible to infer from the signs of the velocity profiles near the transducer (light-blue and purple curves at $t=293.5 \mu \mathrm{s}$ ). However, due to the high viscosity of the simulated fluid, the superimposed motion is quickly dampened down, and, halfway between two transducers, the axial velocity profile has already recovered its original shape (pink and yellow curves at $t=293.5 \mu \mathrm{s})$. During a compression stroke $(t=358.7 \mu \mathrm{s})$, the downwards movement of the transducer surface pumps the surrounding fluid inward and the axial velocity profiles invert their signs. At $t=402.2 \mu \mathrm{s}$ (end of a compression stroke) a situation specular to that observed at $293.5 \mu \mathrm{s}$ is then established. In the same way, at $t=282.6 \mu \mathrm{s}$, something comparable but opposed to that described early at $293.5 \mu \mathrm{s}$ can be observed.
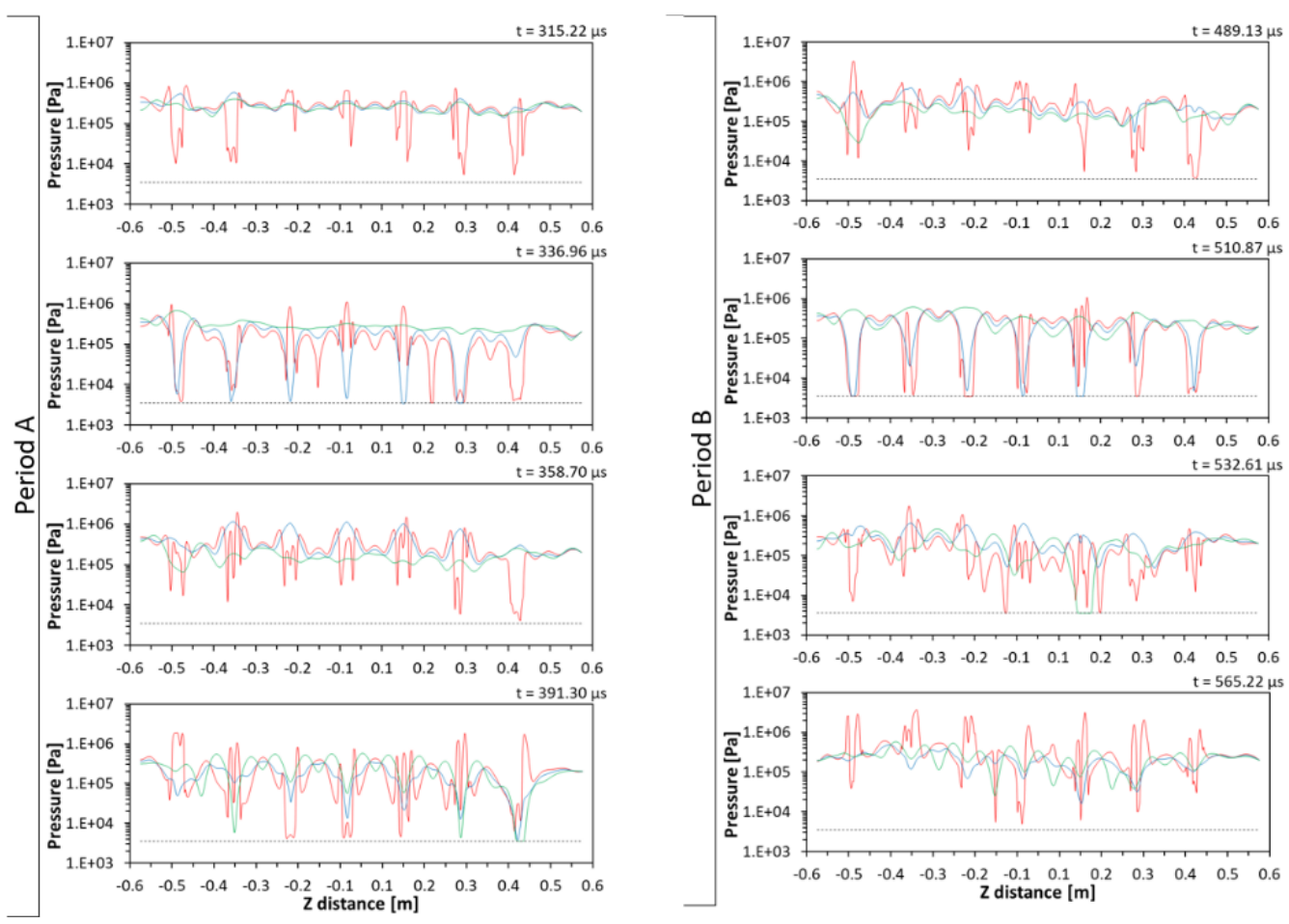

Fig. 11 Comparison between the pressure traces recorded along the three control lines of Fig. 8 and belonging to corresponding instants in two consecutive periods. 


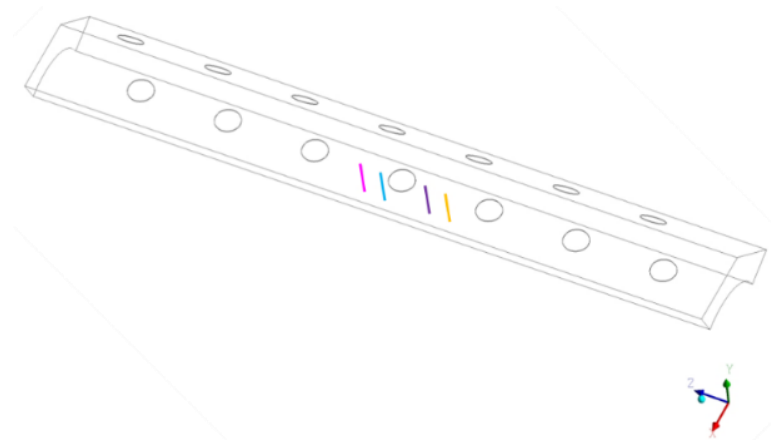

Fig. 12 Location and orientation of the four control lines used in the analysis of the transient simulations for studying the profiles of the velocity components inside the SHE.

The graphs of Fig. 13 (b) shows the presence of a non-negligible circumferential component, practically in each phase of the ultrasound treatment. This result indicates that convective motions are imposed in the non-Newtonian fluid by the vibrating surfaces of the transducers. When $t=282.6 \mu \mathrm{s}$ the transducer surface is at the middle of the expansion stroke and the circumferential velocity shows a "S-type" profile in the vicinities of the transducer. These velocity profiles change sign at about half of the cross-section height. This is also recorded at $t=293.5 \mu \mathrm{s}$, when an expansion stroke ended, and a new compression was starting. This means that in the more external part of the SHE, the flow is going in the opposite direction with respect to that in the inner part of it. At $t=358.7 \mu \mathrm{s}$ and $t=$ $402.2 \mu \mathrm{s}$ the moving surface is at the half and at the end of a compression stroke, respectively. For both of these two cases, a circumferential velocity having comparable magnitude of that of the steady-state case is recorded near the transducer. Between two transducers, a secondary circumferential flow is always present with a magnitude comparable to that of the main flow.

These results highlight that the ultrasound treatment induces non-negligible circumferential motions in the non-Newtonian olive oil paste, giving to this a kind of pulsing swirl movement. It is reasonable to suppose that such pumping movements can promote a secondary mixing effect. Namely, a stirring effect that can result comparable to that imposed during the malaxation step. This effect can enhance the coalescence kinetics, which means that the smaller oil droplets are forced to collide each other to form larger droplets, leading to an increase of oil recovery. The proposed staggered disposition of the transducers was thought for enhancing this beneficial stirring motion. In fact, the constant presence of a circumferential component between transducers suggested that a fluid volume will progress with a slalom path. Due to the pressure globe generated by each transducer, the volume will deflect its trajectory towards a transducer belonging to a parallel row. This velocity component is useful to promote a beneficial stirring process, similar to the malaxer process. 


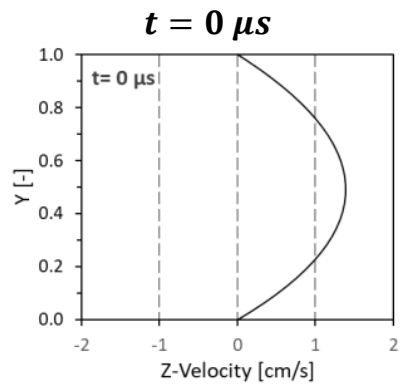

(a)

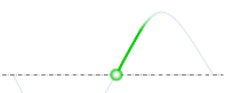

$t=282.6 \mu s$
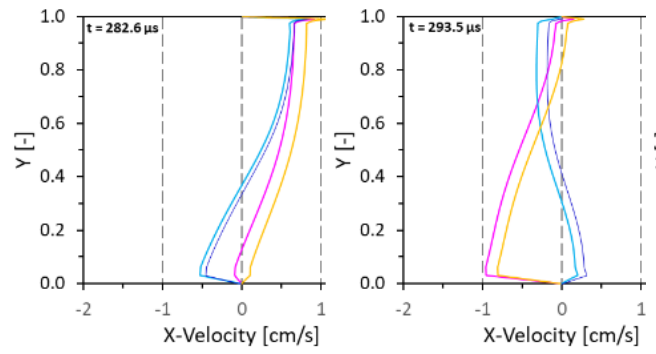

(b)
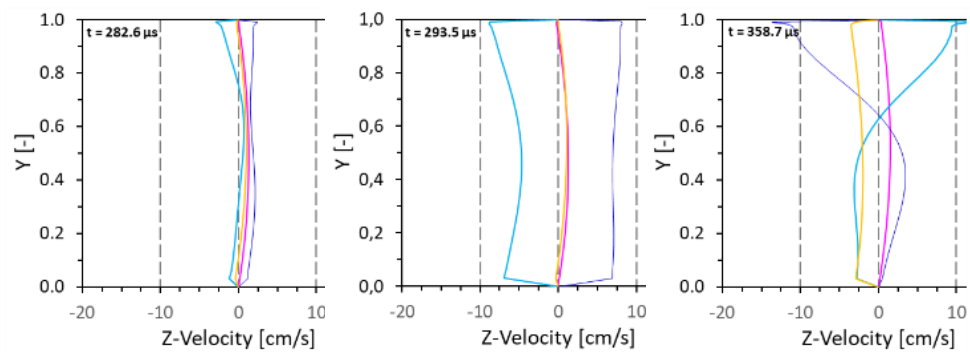

(c)
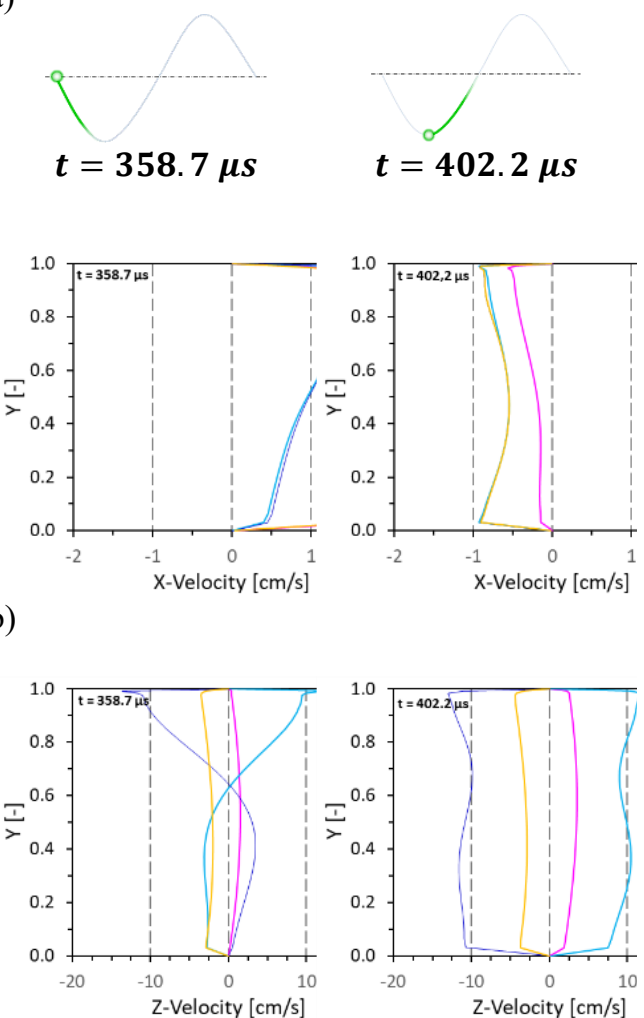

Fig. 13 Undisturbed velocity profiles obtained from the steady-state simulation (a). Profiles of the circumferential (b) and the axial (c) velocities recorded on the four control lines of Fig. 12 and at four different instants. A graphical information about the location of the moving surface at that specific time is also reported for each of the four selected frames.

\subsection{Effect on Extraction Yield}

The experimental tests of the harvest season were performed in four farms (A, B, C, D) from the North to the South of the Apulia Region. The experiments allowed to compare the extraction yield between the traditional and the ultrasound treatment by means of the SonoHeat-Exchanger (SHE). The fruits were harvested during the campaign 2017/2018 by a trunk 
shaker machine and processed $6 \mathrm{~h}$ after the harvesting. The extraction yield (EY EVoo $)$ content was determined following the procedure described in details in a previous work [6]. Olive oil extraction experiments were performed in triplicate.

By comparing the Extra-Virgin Olive Oil (EVOO) samples extracted by means of the traditional and the innovative SHE systems (Table 1), it was possible to observe an average increase of the oil extractability when the latter was employed, and this was recorded in each test. In the same Table the amount of the processed olives is reported for each test, together with the percentage of yields increment. For the best knowledge, it is important to clarify that in the oil mill C (on December 2017) the SHE worked at the $60 \%$ of the maximum power, due to technical problems occurred to the drivers of the ultrasound transducers.

Table 1. Comparison of extraction yields in four olives mills (A, B, C, D) by means of traditional continuous process and ultrasound treatment with SHE, processing four olive cultivars.

\begin{tabular}{|c|c|c|c|c|c|c|}
\hline Miller & $\mathrm{A}$ & $\mathrm{B}$ & $\mathrm{B}$ & $\mathrm{C}$ & $\mathrm{D}$ & $\mathrm{D}$ \\
\hline Cultivar & Coratina & Coratina & Micell. & Peranzana & $\begin{array}{l}80 \% \\
\text { Cor. } \\
20 \% \\
\text { Ogl. }\end{array}$ & $\begin{array}{c}\text { Cellina } \\
\text { di } \\
\text { Nardò }\end{array}$ \\
\hline $\begin{array}{l}\text { Yield traditional } \\
\left(\mathrm{kg}_{\text {evoo }} / 100 \mathrm{~kg}_{\text {olive }}\right)\end{array}$ & $12.1 \%$ & $19.7 \%$ & $14.6 \%$ & $12.4 \%$ & $19.2 \%$ & $13.2 \%$ \\
\hline $\begin{array}{l}\text { Yield US process } \\
\left(\mathrm{kg}_{\text {evoo }} / 100 \mathrm{~kg}_{\text {olive }}\right)\end{array}$ & $17.6 \%$ & $24.1 \%$ & $15.9 \%$ & $12.8 \%$ & $20.3 \%$ & $14.0 \%$ \\
\hline $\begin{array}{c}\% \text { points in } \\
\text { yields increment }\end{array}$ & +5.4 & +4.5 & +1.4 & +0.5 & +1.1 & +0.8 \\
\hline $\begin{array}{l}\text { Olive processed } \\
\text { (tons) }\end{array}$ & 2.6 & 2.6 & 3.9 & 8.0 & 3.7 & 3.0 \\
\hline Harvesting & October & November & November & December & January & January \\
\hline
\end{tabular}

\section{Conclusions}

The present paper shows how to design, realize and test the first continuous ultrasonic full-scale device named Sono-Heat-Exchanger (SHE), based on plate-shape low frequency ultrasonic transducers for the extra virgin olive oil industry. By means of the SHE it was possible to simultaneously increase the work capacity, the extraction yield and quality. The ultrasound technology is able to induce the rupture of cell walls thanks to the cavitation phenomenon, recovering the oil and minor compounds trapped in the uncrushed olive tissue. To design an ultrasound device a full Three-Dimensional (3D) Multiphase Computational Fluid Dynamic (CFD) analysis was proposed and used for the first time in this field of application useful as auxiliary tool. The fluid dynamic analysis was performed to predict the flow path in the ultrasound devices, to evaluate the flow parameters of the olive paste inside the SHE and the cavitation phenomenon. The pressure and vapour fraction calculations are crucial points to setup an optimal design of the cross section of the SHE. The results demonstrated that the pressure drops and velocity fields are suitable to ensure the best ultrasounds effect and a mixing effect very similar to the malaxation process. Calculations in transient conditions highlighted that the pressure waves propagation is strongly affected by the interaction of each transducer and so a $3 \mathrm{D}$ fluid dynamics analysis is necessary to obtain the best results. The cross section of the SHE is designed in order to achieve a reasonable balancing between the need of maximizing the ultrasound effect in the thickness of olive 
paste and the need to avoid the interference with the heat exchanger surface. With a nonappropriate design, an easy and fast damaging of the internal surface of the heat exchanger can occur or, at least, non-adequate effects can be provided to the olive paste.

Many experimental tests have been performed on four real scale mill plants (working overall $24000 \mathrm{~kg}$ of olives during the 2017/2018 campaign), demonstrating how in each experiment oil yields increase and also demonstrating that the SHE effect in terms of yields decreases with the season of the olive oil campaign.

\section{Acknowledgments}

Funding: EU through the Regione Puglia - Progetto: "PRIN CORATINA" cod. P8K5PA8, AGER 2 Project, grant n. 2016-0174; AGER Foundation-Olive Tree and Oil: Competitive - Claims of olive oil to improve the market value of the product; EU project 820587-OLIVE-SOUND - Ultrasound reactor-The solution for a continuous olive oil extraction process H2020-EU.2.1._INDUSTRIAL LEADERSHIP_EIC-FTI-20182020 - Fast Track to Innovation (FTI) - European Union's Horizon 2020 research and innovation program under grant agreement No. 820587.

\section{References}

[1] Chemat F, Khan MK, others. Applications of ultrasound in food technology: processing, preservation and extraction. Ultrason Sonochem 2011;18:813-35.

[2] Chandrapala J, Oliver C, Kentish S, Ashokkumar M. Ultrasonics in food processing. Ultrason Sonochem 2012;19:975-83.

[3] Ashokkumar M. Applications of ultrasound in food and bioprocessing. Ultrason Sonochem 2015;25:17-23.

[4] Patist A, Bates D. Ultrasonic innovations in the food industry: From the laboratory to commercial production. Innov Food Sci Emerg Technol 2008;9:147-54.

[5] Clodoveo ML, Durante V, La Notte D. Working towards the development of innovative ultrasound equipment for the extraction of virgin olive oil. Ultrason Sonochem 2013;20:1261-70.

[6] Clodoveo ML, Moramarco V, Paduano A, Sacchi R, Di Palmo T, Crupi P, et al. Engineering design and prototype development of a full scale ultrasound system for virgin olive oil by means of numerical and experimental analysis. Ultrason Sonochem 2017.

[7] Jiménez A, Beltrán G, Uceda M. High-power ultrasound in olive paste pretreatment. 
Effect on process yield and virgin olive oil characteristics. Ultrason Sonochem 2007; 14:725-31.

[8] Amirante P, Clodoveo ML, Dugo G, Leone A, Tamborrino A. Advance technology in virgin olive oil production from traditional and de-stoned pastes: Influence of the introduction of a heat exchanger on oil quality. Food Chem 2006;98:797-805.

[9] Legay M, Simony B, Boldo P, Gondrexon N, Le Person S, Bontemps A. Improvement of heat transfer by means of ultrasound: application to a double-tube heat exchanger. Ultrason Sonochem 2012;19:1194-200.

[10] Amirante R, Clodoveo ML. Developments in the design and construction of continuous full-scale ultrasonic devices for the EVOO industry. Eur J Lipid Sci Technol 2017;119. doi:10.1002/ejlt.201600438.

[11] Clodoveo ML. Industrial ultrasound applications in the extra-virgin olive oil extraction process: History, approaches, and key questions. Foods 2019;8. doi:10.3390/foods 8040121 .

[12] Bejaoui MA, Beltran G, Aguilera MP, Jimenez A. Continuous conditioning of olive paste by high power ultrasounds: Response surface methodology to predict temperature and its effect on oil yield and virgin olive oil characteristics. LWT-Food Sci Technol 2016;69:175-84.

[13] Jimenez M A, Beltran M G AHMP. Apparatus and method for the continuous heating and uniform ultrasonic treatment of olive paste ES2327308 B1, 2007.

[14] Amirante R, Distaso E, Tamburrano P, Paduano A, Pettinicchio D, Clodoveo ML. Acoustic cavitation by means ultrasounds in the extra virgin olive oil extraction process. Energy Procedia 2017;126:82-90.

[15] Leonardo M, Servilli M, Torricelli G, Cioria F, Pelosi G, Vannini M. Reactor for increasing the quantity of polyphenols and/or the turbidity stability of extra-virgin olive oil, system and method using said reactor. WO2014181284 A1, 2014.

[16] Bellumori M, Cecchi L, Innocenti M, Clodoveo ML, Corbo F, Mulinacci N. The EFSA health claim on olive oil polyphenols: Acid hydrolysis validation and total hydroxytyrosol and tyrosol determination in Italian virgin olive oils. Molecules 2019;24. doi:10.3390/molecules24112179.

[17] Roselli L, Clodoveo ML, Corbo F, De Gennaro B. Are health claims a useful tool to segment the category of extra-virgin olive oil? Threats and opportunities for the Italian olive oil supply chain. Trends Food Sci Technol 2017;68:176-81. doi:10.1016/j.tifs.2017.08.008.

[18] Amirante R, Paduano A. Ultrasound in Olive Oil Extraction. In: Boskou D, Clodoveo 
ML, editors. Prod. from Olive Tree. 2016th ed., InTech; 2016. doi:10.5772/64765.

[19] Clodoveo ML, Hbaieb RH, Kotti F, Mugnozza GS, Gargouri M. Mechanical strategies to increase nutritional and sensory quality of virgin olive oil by modulating the endogenous enzyme activities. Compr Rev Food Sci Food Saf 2014;13:135-54.

[20] Clodoveo ML, Durante V, La Notte D, Punzi R, Gambacorta G. Ultrasound-assisted extraction of virgin olive oil to improve the process efficiency. Eur J Lipid Sci Technol 2013;115:1062-9.

[21] Clodoveo ML, Dipalmo T, Schiano C, La Notte D, Pati S. What's now, what's new and what's next in virgin olive oil elaboration systems? A perspective on current knowledge and future trends. J Agric Eng 2014;45:49-59.

[22] Clodoveo ML. An overview of emerging techniques in virgin olive oil extraction process: strategies in the development of innovative plants. J Agric Eng 2013;44.

[23] Amirante R, Catalano LA, Dadone A, Daloiso VSE. Design optimization of the intake of a small-scale turbojet engine. C - Comput Model Eng Sci 2007;18.

[24] Amirante P, Clodoveo ML, Tamborrino A, Leone A, Paice AG. Influence of the crushing system: phenol content in virgin olive oil produced from whole and destoned pastes. Olives Olive Oil Heal Dis Prev Acad Press London, UK 2010:69-76.

[25] Dymond JH, Malhotra R. The Tait equation: 100 years on. Int $\mathrm{J}$ Thermophys 1988;9:941-51.

[26] Sauer J, Schnerr GH. Unsteady cavitating flow--a new cavitation model based on a modified front capturing method and bubble dynamics. Proc. 2000 ASME fluid Eng. summer Conf., 2000, p. 11-5. 